\title{
Histoire transcendantale/histoire mondaine : de Husserl à Derrida et retour
}

Roberto Terzi

\section{OpenEdition}

\section{Journals}

Édition électronique

URL : http://journals.openedition.org/alter/415

DOI : $10.4000 /$ alter.415

ISSN : 2558-7927

Éditeur :

Association ALTER, Archives Husserl (CNRS-UMR 8547)

\section{Édition imprimée}

Date de publication : 3 décembre 2017

Pagination : 13-32

ISBN : 978-2-9550449-3-3

ISSN : $1249-8947$

\section{Référence électronique}

Roberto Terzi, « Histoire transcendantale/histoire mondaine : de Husserl à Derrida et retour », Alter [En ligne], 25 | 2017, mis en ligne le 01 décembre 2018, consulté le 19 avril 2019. URL : http:// journals.openedition.org/alter/415; DOI : 10.4000/alter.415 


\title{
HISTOIRE TRANSCENDANTALE/HISTOIRE MONDAINE : DE HUSSERL À DERRIDA ET RETOUR
}

\author{
Roberto Terzi
}

La première rencontre de la pensée husserlienne avec la question de l'historicité, de façon exemplaire dans La philosophie comme science rigoureuse, ne semblait pas promettre de longs développements : c'était la possibilité même d'une rencontre réelle qui était mise en question, et ce à cause des éléments constitutifs mêmes de la phénoménologie, à savoir sa démarche eidétique et transcendantale. En tant que domaine de la factualité contingente et variable, milieu de ce qui est déjà donné au sujet, déploiement d'une genèse empirique et réelle, l'histoire pouvait alors paraître incompatible avec le projet d'une philosophie comme science rigoureuse, transcendantale et eidétique. On connaît cependant la transformation et l'élargissement auxquels Husserl soumettra sa propre position, cet élargissement qui s'appuie avant tout sur une prise en compte de la genèse en général et lui permettra aussi d'intégrer de façon plus adéquate l'historicité dans le cadre de sa phénoménologie. Sans vouloir aucunement sous-estimer cet élargissement spectaculaire du transcendantal qui devrait être étudié pour luimême, il faut cependant souligner qu'il s'agit bien d'un élargissement «interne », d'un extraordinaire approfondissement de la position transcendantale qui ne semble pourtant pas mettre en question les deux axes rappelés, à savoir le primat à la fois d'une analyse transcendantale égologique et d'une analyse eidétique. Il suffirait, pour le confirmer, de rappeler deux éléments : d'une part, la Krisis maintient clairement une réduction transcendantale à l'ego unique comme point d'aboutissement de sa démarche, et ce même après avoir introduit la thématique du monde de la vie en tant que monde historique ${ }^{1}$; $\mathrm{d}^{\prime}$ autre

${ }^{1}$ E. Husserl, Die Krisis der europäischen Wissenschaften und die transzendentale Phänomenologie, Husserliana (dorénavant noté Hua) Bd. VI, La Haye, Martinus Nijhoff, 1954 ; trad. G. Granel, La 
part, dans l'Origine de la géométrie Husserl rejette encore l'objection historiciste, en confirmant son ambition de cerner et décrire un « $a$ priori historique », au sens, bien entendu, d'un a priori de l'histoire ${ }^{2}$. L'essentiel pour Husserl semble continuer à consister dans une réduction de l'histoire mondaine qui devrait conduire à une mise en relief des a priori de l'historicité et à sa compréhension à partir des opérations de la subjectivité transcendantale. Ce cadre trouve enfin son autre pièce fondamentale dans le thème de la téléologie, en tant que loi essentielle qui donne l'orientation de l'histoire et s'enracine dans une «intériorité intentionnelle $»^{3}$ laquelle traverse et régit le cours historique.

À partir de ce premier constat nous voudrions poser deux ordres de questions.

1) Une large partie des difficultés théoriques de la position husserlienne, le caractère à la fois séduisant et décevant de ses analyses de l'historicité, les jugements différents que celles-ci peuvent susciter, tout cela ne tient-il pas au fait que l'historicité est précisément le milieu même qui appelle à une complication, voire à une contamination, des rapports entre le transcendantal et le mondain, le transcendantal et l'empirique, l'essence et le fait? Mais aussi, et simultanément, au fait que cette complication ne peut être pensée et dite qu'à partir de l'attitude transcendantale et en passant par elle? Cette contamination sera notre thème, mais, avant d'être notre thème, elle constitue l'une des thèses majeures que l'on peut tirer des premiers textes de Derrida sur Husserl : le mémoire de 1953-1954 sur Le problème de la genèse dans la philosophie de Husserl ${ }^{4}$, la conférence de 1959 "Genèse et structure » et la phénoménologie ${ }^{5}$, I'Introduction à l'Origine de la géométrie de $1962^{6}$. Au fil conducteur de la lecture derridienne de Husserl, il s'agira donc d'explorer quelques-uns des multiples aspects de cette contamination qui appartient à l'historicité.

crise des sciences européennes et la phénoménologie transcendantale, Paris, Gallimard, 1976, §54 et $\S 55$ (dorénavant noté K).

${ }_{2}$ E. Husserl, Die Frage nach dem Ursprung der Geometrie als intentionalhistorisches Problem, in Krisis, Beilage III, p. 382-383; trad. et introduction de J. Derrida, L'origine de la géométrie, Paris, PUF, 1962, p. 207-208 (dorénavant noté OG).

${ }^{3} \mathrm{~K}, \S 15$, p. 74 ; trad. cit. p. 84.

${ }^{4}$ J. Derrida, Le problème de la genèse dans la philosophie de Husserl, Paris, PUF, 1990 (dorénavant noté PG).

${ }^{5}$ J. Derrida, "Genèse et structure » et la phénoménologie, in L'écriture et la différence, Paris, Seuil, 1967, p. 229-251.

${ }^{6}$ J. Derrida, Introduction, in E. Husserl, L'origine de la géométrie, op. cit., p. 3-171 (dorénavant noté IOG). 
2) Nous nous sommes demandé si la contamination en question ne peut au fond être pensée qu'à partir de l'attitude transcendantale ellemême. Il faudrait peut-être ajouter : plus particulièrement, à partir de la transformation phénoménologique du transcendantal, ainsi que des concepts d'essence, d'idéalité, de sens. N'est-ce pas cette transformation elle-même qui implique, en un sens, une complication des oppositions transcendantal/mondain, essence/fait? Ou qui ouvre au moins la voie dans cette direction? Et, s'il en est ainsi, ne pourra-t-on pas trouver chez Husserl lui-même les signes de la contamination dont nous parlons? Chez Husserl lui-même: c'est-à-dire dans ses textes et ses analyses, dans les implications de ses concepts, sinon dans ses intentions.

Dans un parcours en zig-zag, nous poserons la question d'abord par rapport à Derrida (lecteur de Husserl), puisqu'il fait de cette complication ou contamination une thèse explicite, pour revenir ensuite à Husserl : il s'agira alors de solliciter certains de ses textes à la lumière de ces questions et de montrer ainsi que non seulement les analyses de Husserl constituent le point de départ effectif de la radicalisation opérée par Derrida, mais aussi que certains manuscrits, inédits à l'époque des textes derridiens, offrent d'autres pistes pour explorer cette question, même s'il s'agira toujours de pistes problématiques.

Nous serons conduit à aborder trois figures de cette contamination de l'histoire : l'institution, le Fait originaire, l'afflux.

\section{L'institution originaire et l'écriture}

Une partie significative des analyses de Derrida à ce sujet peuvent être lues comme une radicalisation immanente de l'idée husserlienne d'une Urstiftung, d'une institution originaire historique. Dans sa radicalisation génétique de la phénoménologie, Husserl affirme en effet que toute formation de sens historique (et même les idéalités absolues d'une science comme la géométrie) naît d'une Urstiftung, c'est-à-dire qu'elle est entrée « une première fois » dans l'histoire par un acte originaire qui l'a instaurée et par là inaugure une tradition. $\mathrm{Au}$ cours de cette tradition la formation de sens en question demeure disponible, peut être transmise et transformée, oubliée et réactivée. L'expression « une première fois » est de Husserl lui-même et révèle que cette pensée d'une institution originaire vise à rendre compte de façon radicale de l'historicité du sens dans le cadre de la phénoménologie transcendantale, dans une démarche, comme Derrida le souligne, à la fois résolument anti-platonicienne et anti-kantienne: il 
s'agit alors d'élaborer «une question en retour sur le sens le plus originaire selon lequel la géométrie est née un jour <et, $>$ dès lors, est restée présente comme tradition millénaire [...] sur ce sens selon lequel, pour la première fois, elle est entrée dans l'histoire - doit y être entrée $»^{7}$. $C^{\prime}$ est donc cette idée qui donne toute son importance à un texte comme L'origine de la géométrie et qui, par conséquent, se trouve aussi au centre de l'introduction que Derrida écrit pour sa traduction française. Tout au long de son commentaire Derrida (qui, faut-il le rappeler, traduit Stiftung, Urstiftung, stiftende par «fondation », « proto-fondation », « fondateur ») souligne la radicalité de la position husserlienne et l'importance de cette pensée de la Stiftung, en insistant tout particulièrement sur le fait que celle-ci renvoie la genèse des idéalités à la facticité d'une "première fois". Pour Husserl le

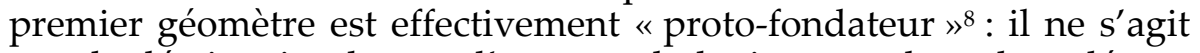
pas de décrire simplement l'acte psychologique par lequel on découvre un sens déjà constitué, mais de montrer la dépendance du sens à l'égard d'un " acte inaugural et fondateur », l'institution d'une formation de sens à partir d'une certaine singularité et facticité, parce que ici "le hic et nunc de la "première fois" est fondateur et créateur »". Et dans les analyses de Husserl, comme on le sait, c'est l'incarnation dans le langage et dans l'écriture qui constitue le moment essentiel de cette Stiftung du sens idéal.

Mais une interrogation traverse, explicitement ou implicitement, tout le commentaire de Derrida : peut-on assumer jusqu'au bout la pensée d'une institution originaire, et avec elle le rôle du langage et de l'écriture, sans mettre en question les principes fondamentaux de la phénoménologie que Husserl voudrait malgré tout conserver ? On peut en effet soulever au moins deux questions concernant la compréhension husserlienne de l'institution originaire : a) en premier lieu, l'institution originaire peut-elle être comprise comme une origine subjective, à savoir comme une activité spontanée de la conscience transcendantale? L'institution originaire relève-t-elle du domaine égologique ou renvoie-t-elle plutôt à une dimension asubjective, à un premier événement historique plutôt qu'à un premier acte historique? b) En deuxième lieu, par son renvoi à une «première fois » du surgissement du sens, l'Urstiftung semble impliquer le rôle d'un événement dans sa facticité et singularité, irréductibles même aux analyses eidé-

\footnotetext{
${ }^{7}$ OG, p. 366 ; trad. cit., p. 175 . Pour une analyse plus détaillée du concept d'institution nous nous permettons de renvoyer à notre essai «Événement, champ, trace : le concept phénoménologique d'institution ", Philosophie, n 131, 2016, p. 52-68.

${ }^{8}$ IOG, p. 23.

${ }_{9}$ IOG, respectivement p. 32 et p. 31 .
} 
tiques les plus fines: ne faut-il pas alors reconnaitre une tension essentielle entre ce thème et la tendance de Husserl à reconstruire une genèse idéelle comprise toujours de façon eidétique?

Même s'il ne contient pas littéralement ces formulations, il nous semble que le texte de Derrida peut être lu à la lumière de ces questions. À l'intérieur du processus de la Stiftung de l'idéalité l'écriture joue un rôle majeur, qui donne aux objectités cette permanence audelà de chaque sujet empirique et déterminable, permanence requise par le statut même de l'idéalité. Précisément pour devenir absolument libres, à savoir valides de façon inconditionnée et omnitemporelle pour tout le monde, les idéalités doivent paradoxalement passer par ce qui, aux yeux d'une démarche classique, apparaîtrait comme une retombée dans l'histoire et dans l'empirie, c'est-à-dire par l'incarnation dans le signe, sans laquelle on n'aurait pas des idéalités, mais des objets psychologiques dans la tête d'un inventeur singulier. Et Derrida de tirer dès maintenant la conséquence décisive de ce paradoxe: "au lieu de l'enchaîner, l'incarnation historique délivre le transcendantal. Cette dernière notion doit donc être repensée » ${ }^{10}$. Si le transcendantal doit être repensé, les questions de la genèse et de l'institution originaire seront alors radicalisées chez Derrida dans la pensée $\mathrm{d}$ 'une contamination originaire et différentielle de l'empirique et du transcendantal, contamination qui constitue le mouvement même de l'historicité11. L'écriture en effet est ici "à la fois événement factice et surgissement de sens ${ }^{12}$, «mouvement de l'incorporabilité essentielle et constituante » et "lieu de l'incorporation factice et contingente ${ }^{13}$ : elle $\mathrm{n}^{\prime}$ achève la constitution des idéalités dans leur permanence qu'en incarnant le sens dans une contingence mondaine. C'est seulement une fois que le sens idéal s'est constitué, qu'il pourra alors faire apparaître en retour son incarnation dans le langage et dans l'écriture comme une extériorisation contingente et inessentielle. Le supplément de l'écriture et de l'incarnation historique produit l'originarité du sens à laquelle elle paraît simplement s'ajouter.

Mais - et il s'agit du pivot même de la stratégie de Derrida, ici comme dans les textes ultérieurs - le passage par l'écriture ne va pas sans conséquences, puisqu'il reste inscrit dans le sens qui est ainsi institué. Le passage par l'écriture inscrit dans le sens la survie au-delà de

\footnotetext{
${ }^{10}$ IOG, p. 71, nous soulignons.

${ }^{11}$ Sur le lien entre la confrontation avec la phénoménologie et la pensée de la trace et de la contamination, cf. l'ouvrage de P. Marrati-Guénoun, La genèse et la trace: Derrida lecteur de Husserl et Heidegger, Dordrecht, Kluwer, 1998.

12 IOG, p. 97.

${ }^{13}$ IOG, p. 90, nous soulignons.
} 
l'auteur qui l'a produit, au-delà de ses intentions vivantes et présentes: l'écriture se montre donc comme le modèle d'un "champ transcendantal sans sujet ${ }^{14}$, à savoir le champ d'une tradition anonyme, constituée comme un texte, qui dépasse en droit les pouvoirs de décision, réactivation, remémoration du sujet. L'écriture inscrit aussi dans le sens la possibilité d'être mal compris ou perdu, jusqu'à la possibilité d'une disparition de la vérité comme conséquence de la possible disparition des archives matérielles dans lesquelles elle est déposée : possibilités extrêmes et toutefois, comme possibilités, ineffaçables et par conséquent constitutives du sens lui-même. En d'autres termes, comme Derrida le dit dans un passage fondamental, l'écriture révèle «le sens transcendantal de la mort ${ }^{15}$ qui est inséparable du sens lui-même. Si l'écriture, instituant l'idéalité, ouvre une tradition de sens, du même coup elle y inscrit la possibilité nécessaire de son échec, exposant le sens à la possibilité de sa perte et du non-sens. Il s'agit d'un type d'argumentation qui revient à plusieurs niveaux dans les textes de Derrida et qui se laisse formaliser ainsi : si la vérité peut disparaître, si le sens peut toujours devenir illisible ou tomber dans l'oubli, si une tradition peut connaître une crise, ces possibilités ne sont pas accidentelles et extérieures par rapport à la vérité, au sens, à la tradition, mais elles sont des possibilités nécessaires qui constituent ces concepts (la vérité doit pouvoir disparaître ou être oubliée ; la tradition doit pouvoir connaître des crises et un oubli, etc.). L'histoire prise au sérieux est le domaine de cette possibilité nécessaire de changement d'un sens prétendu idéal et pur, le seuil de la contamination entre le dedans et le dehors. Et c'est pourquoi, face à la prétention husserlienne de reconstruire une pure histoire interne ou intrinsèque (une innere Historie) ${ }^{16} \mathrm{du}$ sens, et de garder ainsi la distinction entre une histoire transcendantale et une histoire mondaine, Derrida, déjà dans l'Introduction de 1962, peut suggérer que « l'historicité ou l'être-en-histoire, c'est précisément la possibilité d'être intrinsèquement exposé à l'extrinsèque ${ }^{17}$.

\footnotetext{
${ }^{14}$ IOG, p. 84 .

${ }_{15} \mathrm{IOG}$, p. 85.

${ }^{16}$ L'expression se trouve dans OG, p. 386 ; trad. cit., p. 214 ; mais la même idée revient aussi in K, $\S 7$, p. 16 , et $\S 15$ p. 72 ; trad. cit., p. 24 et p. 82 . Comme Derrida le remarque, pour Husserl, même face à l'hypothèse d'une catastrophe de tous les documents écrits dans lesquels la vérité est déposée, le sens de la vérité "garderait sa propre historicité intrinsèque, ses propres enchaînements et la catastrophe de l'histoire mondaine lui resterait extérieure » (IOG, p. 94).

17 IOG, p. 94.
} 


\section{Le Fait et la téléologie}

Les questions de l'Urstiftung et de l'écriture mettent donc en lumière le rôle d'une certaine facticité irréductible dans la genèse historique du sens. On pourrait montrer de différents points de vue que la question de la facticité constitue en effet un fil conducteur qui traverse ces textes de Derrida. Nous nous concentrerons ici sur les dernières pages de l'Introduction, où Derrida soulève cette question à un niveau général et de principe. Une fois acquis le savoir eidétique de la phénoménologie concernant ce qu'est un fait en général et sur le sens en général, donc après le déploiement de l'eidétique phénoménologique,

nous pouvons alors nous demander, en une question qui ne peut plus procéder de la phénoménologie comme telle [...]: "Pourquoi un point de départ factice dans la facticité et une réduction sont-ils possibles en général ?» ou: "Qu'est-ce que la facticité du fait que suppose l'exemplarité du fait? »ou encore: «Qu'est-ce que l'unité originaire du sens et du fait, dont à eux seuls, ni l'un ni l'autre ne peuvent rendre compte $? \gg^{18}$.

La question de la facticité du fait ainsi que celle de l'unité originaire du sens et du fait nous conduisent donc au-delà de la phénoménologie, dans la mesure où l'ambition de celle-ci s'énonce clairement, par exemple, dans cette affirmation du $\S 39$ des Méditations cartésiennes: " le fait et son irrationalité même sont un concept structurel au sein de $\mathrm{l}^{\prime} a$ priori concret ${ }^{19}$. C'est précisément en se référant à ce passage que Derrida peut alors affirmer que, dans une interrogation radicale vers le surgissement de la facticité nue, le Fait sera "toujours plus ou toujours moins, toujours autre, en tout cas, que ce que Husserl le définit $»^{20}$ dans ce texte des Méditations. Derrida vise par conséquent à poser la question d'une facticité du fait qui ne se laisse pas réduire à sa signification phénoménologique et au couple eidos/fait de l'analyse eidétique ; et il faut noter aussi que cette interrogation vers une facticité originaire est également une interrogation vers « l'origine de l'être comme Histoire » ou l'« Être-Histoire »21.

Or il faut constater que Husserl, dans plusieurs manuscrits, en particulier et de façon plus visible à partir des années vingt, a au

\footnotetext{
${ }^{18}$ IOG, p. 168.

${ }^{19}$ E. Husserl, Cartesianische Meditationen und Pariser Vorträge, Hua I, La Haye, Martinus Nijhoff, 1950, §39, p. 114 ; trad. M. De Launay, Méditations cartésiennes et Les conférences de Paris, Paris, PUF, 1994, p. 129.

${ }^{20}$ IOG, p. 169 , note 1.

${ }^{21}$ IOG, p. 169 et p. 170.
} 
moins reconnu ce problème et en a situé le lieu «systématique » par rapport à la phénoménologie. Il s'agit de la perspective de ce que Husserl appelle une "métaphysique », en un sens renouvelé par rapport à celui de la tradition: cette métaphysique est en effet une doctrine du fait et, plus précisément, concerne des faits originaires qui ne peuvent pas être ultérieurement fondés dans ou reconduits à des causes premières, comme cela serait le cas dans une démarche métaphysique traditionnelle. Ainsi, dans Histoire critique des idées, Husserl indique-t-il l'ouverture d'une «problématique qui à son tour n'admet plus d'autre interprétation: celle de l'irrationalité du fait (Faktum) transcendantal qui s'exprime dans la constitution du monde de fait et de la vie spirituelle de fait, c'est donc la métaphysique en un sens nouveau ${ }^{22}$. Husserl réfère ce Fait originaire avant tout à l'ego lui-même et ce qui est remarquable c'est qu'il souligne expressément qu'il se soustrait non seulement à une explication par une cause, mais aussi à l'analyse eidétique de la phénoménologie elle-même. En effet, dans un important manuscrit de 1931, au sujet de l'ego, Husserl écrit: " nous avons là un cas remarquable et unique pour le rapport entre Fait (Faktum) et eidos »; tandis qu'en général l'être des possibilités eidétiques est indépendant de l'être ou non-être de leurs réalisations, « l'eidos Ego transcendantal est impensable sans l'Ego transcendantal en tant que factuel ${ }^{23}$. L'ego qui est à chaque fois est un fait absolu et ineffaçable, ne fût-ce que parce que toute analyse eidétique présuppose l'existence de l'ego et se révèle comme un pouvoir appartenant à sa facticité. Husserl exhibe donc bien un Fait originaire situé en deçà du rapport eidétique habituel fait/essence, lui reconnaît le statut d'un élément dernier et irréductible, le qualifie même comme « l'Absolu » :

l'Absolu a en soi-même son propre fondement et dans son être sans fondement sa nécessité absolue comme la « substance absolue » une. Sa nécessité n'est pas une nécessité d'essence qui laisserait ouvert quelque chose de contingent. Toutes les nécessités d'essence sont des moments de son fait ${ }^{24}$.

C'est la phénoménologie toute entière qui pourrait être repensée à partir de cet arrière-plan "métaphysique » et "factuel», comme le suggère Laszlo Tengelyi dans son dernier ouvrage Welt und Unend-

\footnotetext{
22 E. Husserl, Erste Philosophie (1923-1924). Erster Teil. Kritische Ideengeschichte, Hua VII, La Haye, Martinus Nijhoff, 1956, p. 188 note ; trad. A. Kelkel, Philosophie première (1923-24). Première partie. Histoire critique des idées, Paris, PUF, 1970, p. 269 note.

${ }^{23}$ E. Husserl, Teleologie. Die Implikation des Eidos transzendentale Intersubjektivität im Eidos transzendentales Ich. Faktum und Eidos, in Zur Phänomenologie der Intersubjektivität: Texte aus dem Nachlass. 3, 1929-1935, Hua XV, La Haye, Martinus Nijhoff, 1973, p. 385 (dorénavant noté TI). 24 TI, p. 386.
} 
lichkeit, où il attire l'attention sur cette ouverture husserlienne et essaie de la prolonger, jusqu'à soutenir que par cette idée "la phénoménologie dans l'ensemble de sa construction se montre dépendante de certains faits originaires ou structures de facticité $»^{25}$. Tengelyi repère chez Husserl quatre groupes de faits originaires ${ }^{26}: 1^{\prime}$ ego qui est à chaque fois ; sa possession du monde (Welthabe) ; l'implication intentionnelle de tous les autres ego dans chaque ego de fait; et enfin la téléologie, aussi bien comme téléologie dans la constitution du monde que comme téléologie historique. Plusieurs passages des manuscrits enracinent en effet la téléologie elle-même dans une factualité originaire. Ainsi par exemple, dans le manuscrit déjà cité de 1931, Husserl reconnaît le fait de l'ego, fait allusion aux structures originaires qui appartiennent à sa facticité et qui esquissent la constitution primordiale d'un monde avec sa téléologie, pour tirer enfin la conséquence de sa propre démarche: "dans le Fait est impliqué l'avoir-lieu par avance d'une téléologie. Une pleine ontologie est téléologie, mais elle présuppose le Fait $»^{27}$. Non seulement la téléologie s'enracine dans un Fait originaire, mais, au moins dans un texte, Husserl semble reconnaître que le domaine de l'histoire en général relève de la facticité ou d'un Fait absolu. Il s'agit de l'appendice 32 à Philosophie première. Après avoir évoqué la communauté des monades dans leur implication réciproque et l'avoir qualifiée comme $« l^{\prime} A b s o l u »{ }^{28}$, Husserl conclut :

envisagé en tant qu'absolu, chaque ego a son histoire, et il n'est que comme sujet d'une, de son histoire. Et toute communauté communicative de Je absolus, de subjectivités absolues [...] a son histoire " passive » et "active » et n'est que dans cette histoire. L'histoire est le Fait majeur de l'être absolu (Die Geschichte ist das grosse Faktum des absoluten Seins) ; et les questions dernières, les dernières questions métaphysiques et téléologiques, sont une seule chose avec les $<$ questions $>$ sur le sens absolu de l'histoire ${ }^{29}$.

Si Husserl lui-même semble ici attribuer le statut de facticité au domaine de l'histoire en tant que telle, László Tengelyi, dans l'ouvrage rappelé, essaie de prolonger la perspective husserlienne, en pensant ensemble l'ontologie du monde de la vie et la définition de la méta-

\footnotetext{
${ }^{25}$ L. Tengelyi, Welt und Unendlichkeit : zum Problem phänomenologischer Metaphysik, Munich, Karl Alber, 2014, p. 184.

${ }^{26}$ Cf. ibid., p. $184-185$.

27 TI, p. 385.

${ }^{28}$ E. Husserl, Phänomenologische Reduktion und absolute Rechtfertigung, in Erste Philosophie (1923/24). Zweiter Teil. Theorie der phänomenologischen Reduktion, Hua VIII, La Haye, Martinus Nijhoff, 1959, p. 505.

${ }^{29}$ Ibid., p. 506. Ce texte a fait l'objet d'un commentaire de la part de L. Landgrebe, « Méditation sur le mot de Husserl : "L'histoire est le fait majeur de l'être absolu" », trad. G. Fagniez, in Philosophie, $\mathrm{n}^{\circ} 110,2011$, p. 31-45.
} 
physique comme doctrine des faits originaires: il s'agirait alors d'élargir le champ de cette facticité à toute l'expérience du monde de la vie, en tant qu'expérience de formations de sens (Sinnbildungen) autonomes, événementielles, historiques et factuelles. Ces expériences du monde de la vie, qui se trouvent au fond des institutions de sens (Sinnstiftungen) scientifiques, ne peuvent être réveillées que dans l'après-coup, par une question en retour (Rückfrage): question en retour qui se déploie à partir de ce qui est déjà institué vers la formation de sens spontanée sous-jacente, ainsi qu'à partir de la corrélation modes de conscience/modes de donation vers l'ontologie qui la soutient ${ }^{30}$. Comme Derrida déjà le soulignait, la question en retour n'a pas qu'une signification méthodologique extrinsèque, mais est le signe même de l'historicité et de la facticité de la pensée comme retard ${ }^{31}$.

Nous sommes ainsi conduits à une possible «fondation » factuelle du thème téléologique et transcendantal : la téléologie s'enracine dans un fait originaire qui est en deçà du couple fait-essence et ne peut être ultérieurement fondé ou interrogé quant à son «pourquoi »; plus précisément, elle s'enracine dans ce «réseau» de faits originaires représentés par l'ego lui-même et son implication intentionnelle avec une communauté de monades. À la lumière de ce réseau de faits originaires qui ont leur pivot dans l'ego, quelle est la nature de ce fait dernier qui se trouve au fond de la téléologie? La réponse de plusieurs textes de Husserl renvoie à une intentionnalité ou force pulsionnelle qui caractérise la vie de la subjectivité transcendantale comme telle et ne peut pas être ultérieurement expliquée : ainsi dans le manuscrit C13 Husserl parle-t-il d'un « instinct transcendantal - en un sens la tendance universelle qui traverse la totalité de l'intentionnalité de l'ego - la téléologie universelle permanente ${ }^{32}$. Cette force pulsionnelle est la tension de la vie à se réaliser pleinement, jusqu'au niveau d'une intersubjectivité intergénérationnelle: «ce processus téléologique, le processus d'être de l'intersubjectivité transcendantale, porte en soi une "volonté de vie" universelle [...]»33. Il s'agit par conséquent aussi de la force qui historiquement oriente l'humanité vers la réalisation progressive des Idées comme buts situés à l'infini

\footnotetext{
${ }^{30}$ Cf. L. Tengelyi, Welt und Unendlichkeit, op. cit., p. 213-227.

${ }^{31}$ Cf. IOG, p. 20, p. 35-36, p. 170.

32 E. Husserl, Späte Texte über Zeitkonstitution (1929-1934): Die C-Manuskripte, Hua Materialen Bd. VIII, Dordrecht, Springer, 2006, p. 260.

33 TI, p. 378.
} 
et on comprend alors que la Krisis évoque une «puissance instinctive (Triebkraft) ${ }^{34}$ de la tâche téléologique qui traverse notre histoire.

Cet entrelacs entre Fait, téléologie et force pulsionnelle aurait d'une part l'avantage de mettre en lumière la cohérence interne de la phénoménologie husserlienne, en montrant au niveau le plus radical que la téléologie n'est pas une vision idéologique de l'histoire superposée à d'autres analyses pour des raisons historiques contingentes, mais bel et bien le prolongement au niveau de l'histoire d'une structure de l'expérience qui pour Husserl caractérise la vie et l'intentionnalité comme telles. D'autre part, la position de Husserl garde cependant des aspects problématiques concernant la question de l'historicité. En premier lieu, on peut en effet se demander ce qu'il en est de la spécificité et de l'originalité du domaine de l'historicité au moment où il serait compris comme le prolongement d'une force pulsionnelle qui caractérise la vie comme telle. En second lieu, il s'agirait de soulever la question de savoir si cette «identification » du Fait originaire avec une pulsion orientée téléologiquement ne constitue pas déjà une transgression des limites de la «métaphysique » comme doctrine de la factualité originaire. Par ailleurs, si tout le mouvement vital et historique est régi par cette pulsion ou volonté qui, comme Husserl le dit ${ }^{35}$, tend vers la «perfection infinie » et l'autoréalisation de la vie, vers l'infinité et l'éternité, on ne s'étonnera peut-être pas du fait que cette téléologie débouche sur une perspective théologique, quoique en un sens tout à fait particulier. Husserl affirme en effet que «la volonté absolue universelle, qui vit dans tous les sujets transcendantaux $[. .$.$] est la volonté divine »^{36}$, pour se demander enfin : «Peut-on dire, dans cette situation, que cette téléologie, avec son archi-facticité, aurait son fondement en Dieu ? $»^{37}$. En troisième lieu, et plus généralement, si on garde à l'esprit tous les autres textes plus connus qui décrivent la téléologie historique, le dévoilement de son caractère archi-factuel ne semble pas modifier, aux yeux de Husserl, le statut de cette téléologie, à savoir sa solidité, sa plénitude, sa valeur transcendantale, constitutive et essentielle par rapport à l'histoire mondaine. En d'autres termes, et pour reprendre la façon dont Derrida a parfois exploité les significations de ce mot français,

\footnotetext{
${ }^{34} \mathrm{~K}, \S 15$, p. 72 ; trad. cit., p. 82. Cf. D. Pradelle, Généalogie de la raison. Essai sur l'historicité du sujet transcendantal de Kant à Heidegger, Paris, PUF, 2013, p. 145-146.

${ }_{35}$ Cf. TI, p. 379.

36 TI, p. 381.

${ }^{37}$ TI, p. 385 . Cf. aussi K, p. 7 ; trad. cit., p. 14 : « Le problème de Dieu contient manifestement le problème de la raison "absolue" comme source téléologique de toute raison dans le monde, le problème du "sens" du monde ".
} 
Husserl ne semble pas prendre en compte que la préséance de ce Fait « entame» la téléologie au double sens du verbe «entamer»: il inaugure la téléologie, l'institue, pourrait-on dire, et par là même il l'incise, la compromet, porte atteinte à sa plénitude et à son prétendu statut purement transcendantal, empêchant à jamais qu'elle puisse être pure. Ce qui revient à dire que, dans tous les sens de l'expression, la contamination est à l'origine.

Cette argumentation derridienne est contenue en germe déjà dans Le problème de la genèse et précisément aussi au sujet de la téléologie. Dans l'avertissement écrit en 1990 pour la publication de son mémoire de 1953-1954, Derrida affirme en effet y retrouver les premiers signes d'un geste et d'un questionnement qui resteront ensuite constants dans son œuvre: il s'agit toujours " d'une complication originaire de l'origine, d'une contamination initiale du simple, d'un écart inaugural qu'aucune analyse ne saurait présenter, rendre présent dans son phénomène $»^{38}$. Le texte de 1953-1954 essaie de développer cette thèse en relisant tout le parcours de Husserl au fil conducteur de la question de la genèse, pour l'« appliquer » enfin, pour ainsi dire, au domaine de l'histoire et en particulier à la question de l'Idée téléologique au sens kantien. Aux yeux de Derrida la téléologie apparaît déjà, en tant que telle, comme une façon d'escamoter la contamination de l'histoire : si la phénoménologie génétique conduit Husserl à découvrir une genèse passive qui est en deçà de l'activité intentionnelle du sujet, si cette passivité qui entoure et précède l'ego est aussi le résultat de la sédimentation historique, la téléologie s'avère alors comme un moyen de maîtriser ce domaine en lui redonnant un sens intentionnel. Autrement dit, la genèse passive réintroduit le constitué dans la sphère transcendantale constituante et conduit aussi à dévoiler l'homme originairement pris dans son environnement historique : pour sauver l'idéalisme transcendantal il fallait que cette passivité soit animée par une intentionnalité supra-subjective, qui la transforme de passivité inerte en projet pré-conscient et pré-actif ${ }^{39}$. C'est exactement le rôle de la téléologie, qui permet à Husserl de « réapproprier, en l'essentialisant et en en prescrivant en quelque sorte l'horizon, une genèse sauvage qui devenait de plus en plus

\footnotetext{
38 PG, p. VI-VII. Ou, en d'autres termes, la question est celle déjà formulée dans le texte originel de 1953-1954: "comment l'originarité d'un fondement peut-elle être une synthèse a priori ? Comment tout peut-il commencer par une complication? » (PG, p. 12).

39 Pour ce qui précède, cf. PG, p. 247-248.
} 
envahissante et qui semblait s'accommoder de moins en moins de l'apriorisme phénoménologique et de l'idéalisme transcendantal ${ }^{40}$.

La téléologie devrait ainsi sauver le projet phénoménologique, mais pour accomplir cette tâche elle devrait garder sa priorité et sa pureté par rapport à l'histoire mondaine. L'Idée téléologique devrait rester «imperturbable et incorruptible ${ }^{41}$, mais alors

comment l'idée téléologique peut-elle être démentie, ignorée, pervertie ou dissimulée, inexistante ou « oubliée » pour certains sujets, à certains moments de l'histoire [...] ? Comment la « crise » d'une idée téléologique transcendantale est-elle possible? Comment l'événement empirique, s'il est seulement constitué, peut-il « recouvrir » et « voiler » 1'acte même de sa constitution ${ }^{42}$ ?

Ce constat oblige alors à compliquer le cadre des distinctions husserliennes, comme Derrida le fait par une question évidemment rhétorique: "Est-ce que la simple existence de "crises" ne nous obligera pas à faire descendre dans une histoire "mondaine" l'idée de la philosophie et à prêter un rôle constituant à l'événement empirique lui-même ? ${ }^{43}$. La prise en compte effective de l'historicité requiert donc d'attribuer un rôle constituant à l'événement empirique, ce qui conduit à reconnaître que la crise n'est pas un accident, mais une "nécessité interne de $l^{\prime}$ histoire ${ }^{44}$. S'en tenir à la seule activité intentionnelle de la subjectivité comme source de sens et à une séparation étanche entre le transcendantal et le mondain signifie s'interdire de rendre compte $\mathrm{du}$ mouvement effectif de l'histoire. D'ailleurs, si l'Idée ne peut pas rester pure au cours de son histoire, c'est parce que, suivant Husserl, elle n'est pas une idée "platonicienne", mais un événement historique, l'événement de son institution originaire : elle a eu une genèse dans l'histoire, elle naît d'une synthèse originaire avec une situation historique mondaine qui la précède et elle est donc « impure », contaminée, dès sa naissance. Et, comme Derrida l'écrit, «si l'idée n'est pas originairement absolue [...] elle ne le sera jamais $»^{45}$; phrase qui d'ailleurs, à la lumière d'autres textes derridiens, pourrait aussi être renversée en "si l'idée se révèle comme non-absolue, c'est qu'elle ne l'a jamais été ». Le caractère factuel de l'Idée et donc (dans la perspective de Husserl) de la philosophie elle-

\footnotetext{
40 J. Derrida, «Genèse et structure » et la phénoménologie, op. cit., p. 232. Cf. aussi PG, p. 39-40, p. $240-241$.

${ }_{41}$ PG, p. 248.

42 PG, p. 248-249.

43 PG, p. 249.

${ }_{44}$ PG, p. 274.

${ }^{4}$ PG, p. 272-273.
} 
même résiderait alors non pas dans une force pulsionnelle orientée téléologiquement, mais dans le Fait historique, non-fondé, de son institution originaire ; et puisqu'elle naît d'une institution originaire, l'Idée d'une tradition est toujours déjà différente de soi, en un sens elle est toujours déjà en crise ou, pourrait-on dire, en déconstruction.

\section{L'afflux du transcendantal dans le mondain}

Mais une complication des rapports entre l'historicité transcendantale et l'historicité mondaine ne serait-elle pas au fond exigée par le sens même du transcendantal phénoménologique? Dans La voix et le phénomène, Derrida souligne la spécificité du rapport entre le transcendantal et le mondain en phénoménologie, celle qu'on pourrait appeler leur différence "non réelle» : aucune différence déterminée ne distingue le transcendantal et le mondain, qui restent pourtant séparés par un saut radical, et ce qu'il faut penser est alors précisément «ce rien qui distingue des parallèles » ${ }^{46}$. Le transcendantal n'est pas «quelque chose » d'autre par rapport au mondain, n'étant rien d'autre que son sens, et « cette duplication du sens ne doit correspondre à aucun double ontologique $\gg^{47}$. Si, comme Husserl luimême le souligne, dans leur différence irréductible le Je transcendantal et le Je mondain sont le même, ne serait-on pas en droit d'étendre cette proposition au rapport entre histoire transcendantale et histoire mondaine? En outre, précisément parce que la philosophie, comme instauration du transcendantal et de l'Idée, s'institue au sein d'une histoire, elle peut en même temps y faire retour. Il s'agit d'une autre direction de la complication des rapports concernant l'histoire, que l'on peut repérer chez Husserl, la direction dans laquelle il semble peut-être aller le plus loin et qui s'exprime dans le concept de Einströmen (" afflux», " affluer»)4. Celui-ci revient dans plusieurs passages de la Krisis et des manuscrits contemporains comme un terme technique, doué d'une signification relativement déterminée. Il apparaît en particulier dans le titre même du $\S 59$ de la Krisis, qui, à propos des rapports entre la psychologie et la phénomé-

\footnotetext{
${ }^{46}$ J. Derrida, La voix et le phénomène, Paris, PUF, 1967, p. 12.

47 Ibid., p. 11.

${ }^{48}$ Nous reprenons la traduction adoptée par N. Depraz, tandis que G. Granel, dans la Krisis, traduit de plusieurs façons, mais notamment par "entrer-dans-le-flux ». Pour les raisons que nous verrons dans un instant, eu égard au mouvement indiqué par ce concept, une traduction plus « explicitante » ou interprétative pourrait être « refluer» (comme dans certains passages de la traduction italienne de la Krisis par E. Filippini, Milano, Net, 2002).
} 
nologie, évoque «le problème de l'“afflux" (des "Einströmens") ». La réduction transcendantale consiste dans cette suspension de l'attitude naturelle par laquelle je me découvre comme ego transcendantal, cet ego dont l'homme psycho-physique n'est qu'une objectivation. Mais « je suis en tant qu'ego transcendantal cela même qui, dans la mondanité, est un ego humain $»^{49}$ : par conséquent, non seulement mon ego transcendantal s'est toujours déjà auto-objectivé comme ego mondain, mais moi en tant que phénoménologue je peux toujours, et de fait je dois toujours, revenir de l'attitude transcendantale à l'attitude naturelle, à ma vie mondaine et quotidienne. Dès lors « l'ancienne objectivation naïve de soi en tant que "je" empirique humain de ma vie psychique se voit prise dans un mouvement nouveau » : en effet tout ce que j'ai obtenu grâce à la réduction n'est pas perdu, mais «afflue (strömt...ein) maintenant dans l'objectivation-de-soi, dans la vie psychique $»^{50}$. Les acquis transcendantaux se mondanisent avec moi, se localisent dans mon âme et dans le monde. D'où le statut du phénoménologue et le changement de regard que la phénoménologie porte avec soi dans ce mouvement :

en tant que phénoménologue je puis certes à tout moment revenir à l'attitude naturelle, au simple accomplissement de mes intérêts vitaux $[\ldots]$. Comme avant - et cependant pas tout à fait comme avant. Car l'ancienne naïveté, je ne peux plus l'atteindre, je ne peux plus que la comprendre ${ }^{51}$.

Einströmen indique donc la mondanéisation du transcendantal, au sens précis de l'afflux et de l'incorporation des aperceptions transcendantales dans le monde «naturel » ou, en d'autres termes, au sens du «refluer» des acquis de la phénoménologie transcendantale (de moi comme phénoménologue) dans l'attitude naturelle. Ce mouvement implique évidemment aussi une signification historique, puisqu'il concerne d'une part l'enrichissement du monde par l'incorporation d'une production historique (la philosophie), d'autre part l'insertion de la philosophie dans l'histoire ${ }^{52}$. Or cette même problé-

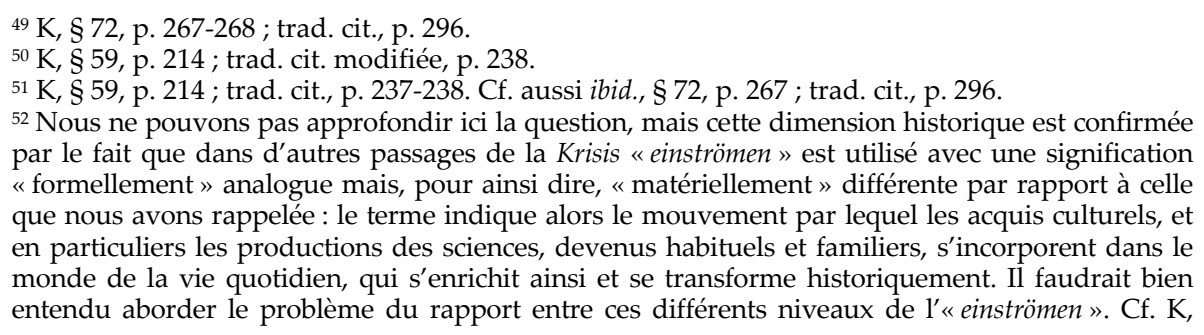


matique est aussi l'objet d'un remarquable et dense manuscrit de 1935, intitulé précisément Einströmen ${ }^{53}$, qui a l'intérêt d'expliciter davantage la dimension historique de $\mathrm{l}^{\prime}$ «afflux ». Par le mouvement de l'afflux en effet

le monde lui-même se transforme [...] il se transforme par cette mondanéisation du transcendantal, qui ainsi - dans cette transformation - est tout à fait absorbé en lui. Le mouvement de cette transformation a la signification constitutive d'une création transformatrice (Umschöpfung) du monde, qui commence avec la mise en place de la réduction ${ }^{54}$.

Le monde se transforme par rapport à son sens naturel et se constitue maintenant de façon mobile dans le mouvement du «pouvoir effectuer toujours à nouveau des nouveaux dévoilements transcendantaux » et $\mathrm{du}$ " pouvoir revenir toujours à nouveau dans l'attitude mondaine » : le monde reçoit ainsi un nouveau sens, " qui implique $a$ priori tout transcendantal, tout [transcendantal] dans le mouvement du déjà mondanisé [...], et tout [transcendantal] en tant qu'il peut être interrogé quant à son historicité transcendantale, quant à son êtredevenu transcendantal et à son devenir transcendantal $»^{55}$.

Cette historicité du transcendantal dans son mouvement a lieu dans une temporalité transcendantale qui est «double» et "corrélative »: il s'agit de la corrélation entre la temporalité de l'intersubjectivité transcendantale et celle du monde, où la deuxième est incluse comme corrélat dans la première (selon un schéma husserlien plus «classique»), mais «la première aussi est, d'une autre façon, dans la deuxième en tant qu'affluée et affluante et elle doit être montrée et parcourue par une question en retour transcendantale $»^{56}$. L'historicité de l'intersubjectivité transcendantale afflue donc dans le monde et le monde à son tour a tendance à garder «sa propre forme catégoriale $»^{57}$ : dans ce mouvement de l'afflux le monde garde ou reconstitue sa forme catégoriale de "tout de l'étant », mais cela implique désormais qu'il inclut et absorbe en soi aussi le transcendantal qui se mondanéise. Par la réduction on découvre que l'ancien monde « naturel » n'était pas véritablement le «tout de l'étant», le monde se

p. 115 , p. 141 note, p. 213, p. 466 ; trad. cit. p. 129 (traduit par « se mêler à »), p. 157 note (« venir se jeter »), p. 237 (« entrer dans le flux»), p. 517-518 (« confluer»).

${ }^{53}$ E. Husserl, Einströmen, in Die Krisis der europäischen Wissenschaften und die transzendentale Phänomenologie, Ergänzungsband, Hua XXIX, Dordrecht, Kluwer, 1993, p. 77-83.

54 Ibid., p. 79.

${ }^{5}$ Ibid., p. 80.

${ }^{56}$ Idem.

${ }^{57}$ Ibid., p. 78 ; cf. aussi p. 81, dont nous résumons et simplifions le développement dans les lignes suivantes. 
transforme et devient maintenant la véritable totalité en absorbant en soi aussi le transcendantal mondanéisé qui reflue en lui.

Résumons: la réduction transcendantale implique une rupture avec l'attitude naturelle et l'histoire mondaine pour ouvrir le domaine d'une historicité transcendantale, mais le phénoménologue fait retour dans l'attitude naturelle et par là les résultats transcendantaux affluent dans le monde et dans son histoire. La phénoménologie prend sa place dans l'histoire mondaine et la modifie par la conscience qu'elle porte avec soi de son regard : je peux revenir à ma vie précédente pour comprendre ce qu'était ma naïveté. Il importe de souligner deux aspects essentiels concernant le sens de cet afflux.

1) Pour Husserl ce mouvement n'indique pas un simple événement subjectif (au sens le plus étroit du terme : un fait qui concernerait le seul phénoménologue en tant qu'individu et le changement de son rapport personnel au monde), mais un véritable événement constitutif: la constitution du monde se poursuit (et le monde assume ainsi, comme nous avons vu, un sens nouveau) à travers cette mondanéisation et cet afflux du transcendantal, à travers l'œuvre de la phénoménologie, qui participe ainsi à l'histoire du monde. La Krisis le dira clairement: la recherche transcendantale elle-même "est un procès historico-mondain, dans la mesure non seulement où elle enrichit d'une nouvelle science l'histoire de la constitution du monde, mais aussi où elle enrichit pour tous et chacun le contenu du monde $»^{58}$.

2) Avec ces questions nous nous trouvons évidemment au niveau d'une "phénoménologie de la phénoménologie » et d'une réflexion sur sa méthode. En indiquant la mondanéisation «consciente » des acquis de la phénoménologie transcendantale, ou, en d'autres termes, la mondanéisation du spectateur transcendantal, le concept husserlien de Einströmen correspond à ce que Fink dans la Sixième Méditation cartésienne appelle "mondanéisation secondaire » ou "impropre » (distincte de la «mondanéisation primaire » comme auto-objectivation du sujet transcendantal dans l'homme ${ }^{59}$. Face à la tendance de son assistant à souligner l'écart voire l'abîme de sens entre le plan de la vérité transcendantale et celui de la vérité mondaine - tendance qui aboutira à la définition de la deuxième comme "vérité apparente »Husserl écrit un commentaire extrêmement significatif pour notre problème : «dans l'ensemble de l'exposition, il faut éviter l'apparence

\footnotetext{
${ }_{58} \mathrm{~K}, \S 72$, p. 268 ; trad. cit., p. 296 , nous soulignons.

${ }^{59}$ Cf. E. Fink, VI. Cartesianische Meditation. Teil 1. Die Idee einer transzendentalen Methodenlehre, Hua Dokumente Bd. II/1, Dordrecht, Kluwer, 1988 ; trad. N. Depraz, Sixième méditation cartésienne. Première partie. L'idée d'une théorie transcendantale de la méthode, Grenoble, Jérôme Millon, 1994, § 11b : «La mondanéisation de l'activité phénoménologisante ».
} 
selon laquelle la mondanéisation de la phénoménologie [...] serait une mauvaise chose qui ne donne occasion qu'à des erreurs, à des malentendus »; par la réduction transcendantale l'homme gagne en effet « la possibilité d'une humanité nouvelle, plus haute », mais cette possibilité inclut le retour dans le monde pour le considérer comme corrélat et y «trouver objectivés historiquement tous les acquis transcendantaux». Ce que l'homme gagne par là est donc du même coup « la possibilité d'une existence mondaine nouvelle par la connaissance transcendantale de soi », par le fait que «tout le transcendantal est rétroprojecté dans la mondanéité ». Dans le retour à l'attitude naturelle "le monde lui-même reçoit alors pour lui une nouvelle dimension à même le transcendantal qui afflue en lui ». Or ce monde « en lequel le transcendantal dévoilé a afflué et continue d'affluer [...] n'est pas pour lui simplement le monde au sens commun [...] du terme, à quoi s'ajoute une élucidation transcendantale, mais il est pour lui un monde d'un sens mondain nouveau, et sa vie dans le monde $[\ldots]$ a un style mondain nouveau $»^{60}$.

«Une existence mondaine nouvelle», «un sens mondain nouveau »: toute l'énigme se concentre peut-être dans ces expressions, par lesquelles Husserl semble ouvrir une nouvelle dimension pour la phénoménologie. "L'histoire du monde » qui se modifie et s'enrichit dans le mouvement de l'afflux n'est plus simplement une histoire mondaine au sens d'une histoire constituée, qui devrait être mise entre parenthèses et reconduite à sa source transcendantale-subjective. En effet, quel est le statut de ce monde, qui est d'un côté le monde de l'attitude naturelle, mais, de l'autre côté, inclut en soi le transcendantal ? Et quel est le statut de cette historicité qui inclut à la fois l'historicité transcendantale et l'historicité mondaine, l'historicité transcendantale en tant qu'elle reflue dans l'historicité mondaine? Cette historicité qui consiste dans la possibilité de passer toujours à nouveau du transcendantal au mondain et réciproquement? Force est de constater que, à la rigueur, nous sommes ici face à un sens du monde qui se soustrait à la dichotomie du monde «naturel » et du monde comme corrélat de la subjectivité transcendantale; que nous sommes face à un sens de l'historicité qui est au-delà du partage de la pure historicité transcendantale constituante et de l'historicité mon-

\footnotetext{
${ }^{60}$ Ibid., commentaire de Husserl, p. 143, note 469 ; trad. cit., p. 187, note 469, souligné dans le texte. Pour une confrontation entre les positions de Husserl et Fink, que nous ne pouvons pas effectuer ici, cf. S. Luft, "Phänomenologie der Phänomenologie ». Systematik und Methodologie der Phänomenologie in der Auseinandersetzung zwischen Husserl und Fink, Dordrecht, Kluwer, 2002, en particulier p. 230-288, où l'auteur définit le concept de Einströmen comme le «Gegenkonzept » husserlien par rapport à la «mondanéisation secondaire » de Fink (p. 265).
} 
daine constituée. La «situation dernière » à laquelle l'analyse de Husserl nous renvoie est celle d'une historicité et transcendantale et mondaine, ni transcendantale ni mondaine, parce qu'elle n'est que le mouvement du passage et de la différence d'un plan à l'autre. Et c'est aussi à cette situation que l'on pourrait alors référer, mutatis mutandis, les deux célèbres phrases par lesquelles se termine l'Introduction à l'Origine de la géométrie de Derrida : "l'Absolu est le Passage ", "transcendantale serait la Différence $»^{61}$.

En quoi consisterait la modification que la phénoménologie apporte dans l'histoire du monde en y affluant? Il ne s'agit évidemment pas d'un simple fait historique intramondain qui changerait le cours de l'histoire, mais d'un changement du regard. Ce que ce mouvement apporte, $c^{\prime}$ est précisément la compréhension que le monde dans lequel nous sommes "naturellement ", que nous assumons comme donné et allant de soi, peut être interrogé quant à sa formation, c'est-à-dire quant à sa genèse historique, et analysé en tant que résultat d'un mouvement historique constituant ou plutôt instituant. Dès lors l'instance qui « constitue », qui peut rendre compte de la formation de l'histoire dans l'enchevêtrement et la sédimentation de ses couches, ne serait pas une «subjectivité » transcendantale, mais, pour reprendre une autre expression de Husserl lui-même, une «vie transcendantale » : une vie transcendantale anonyme dans l'implication infinie de ses différentes opérations intentionnelles, une "vie opératoire universelle ${ }^{62}$ qui fonctionne constamment de façon anonyme et passive, intersubjective et historique. Si l'analyse de la genèse historique reconduit en dernière instance à des institutions (Stiftungen), on pourrait parler d'une «vie instituante » : une vie qui se réalise dans les différentes institutions créatrices de formations de sens historiques ${ }^{63}$.

Toute histoire mondaine peut donc être l'objet d'une question en retour sur sa provenance à partir d'un réseau d'institutions originaires appartenant à cette vie anonyme. Cette question en retour exhibera alors la contamination originaire de la facticité et du sens

\footnotetext{
${ }^{61}$ IOG, p. 165 et p. 171.

${ }^{62} \mathrm{~K}, \S 38$, p. 148 ; trad. cit., p. 165.

${ }^{63}$ Nous reprenons en la modifiant une suggestion de B. Bégout, qui dans L'enfance du monde, Chatou, Les Éditions de la Transparence, 2007, p. 136, évoque une " production transcendantale » pour rendre compte de la "puissance constituante de la vie transcendantale » (distincte de la subjectivité transcendantale) et donc de la formation du monde de la vie.
} 
(puisque la facticité est impliquée par le concept d'institution et concerne l'ensemble du domaine historique), ainsi que celle du transcendantal et du mondain (car le premier afflue et a toujours déjà afflué dans le deuxième, constituant une historicité "transcendantale-mondaine»). C'est ce que nous avons essayé de montrer à partir de Derrida: l'historicité se présente comme le domaine même d'une contamination irréductible du transcendantal et de l'empirique, du transcendantal et du mondain, de l'essentialité et de la factualité autrement dit, l'historicité est ce qui a toujours déjà défait la pureté et la pertinence absolues de ces oppositions. La vie opératoire et instituante est donc aussi instituée : toujours déjà prise dans une facticité irréductible, marquée par le fait du langage dans lequel elle doit s'exprimer, dépassée par ce qu'elle institue et qui lui survit comme une trace - toujours déjà impliquée et refluée dans le monde. Ce n'est peut-être que par cette voie que l'on pourra rendre compte de cette définition de l'histoire : "l'histoire n'est d'entrée de jeu rien d'autre que le mouvement vivant de la solidarité et de l'implication mutuelle (des Miteinander und Ineinander) de la formation de sens (Sinnbildung) et de la sédimentation de sens originaires ${ }^{64}$.

${ }^{64}$ OG, p. 380 ; trad. cit., p. 203. 